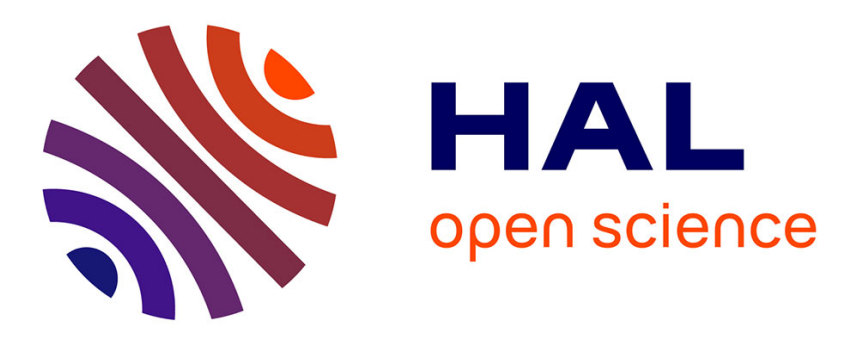

\title{
An inversion approach for analysing the physical properties of a seismic low-velocity layer in the upper mantle
}

Jie Xiao, Saswata Hier-Majumder, Benoit Tauzin, Dave Waltham

\section{- To cite this version:}

Jie Xiao, Saswata Hier-Majumder, Benoit Tauzin, Dave Waltham. An inversion approach for analysing the physical properties of a seismic low-velocity layer in the upper mantle. Physics of the Earth and Planetary Interiors, 2020, 304, pp.106502. 10.1016/j.pepi.2020.106502 . hal-03025198

\section{HAL Id: hal-03025198 \\ https://hal.science/hal-03025198}

Submitted on 7 Dec 2020

HAL is a multi-disciplinary open access archive for the deposit and dissemination of scientific research documents, whether they are published or not. The documents may come from teaching and research institutions in France or abroad, or from public or private research centers.
L'archive ouverte pluridisciplinaire HAL, est destinée au dépôt et à la diffusion de documents scientifiques de niveau recherche, publiés ou non, émanant des établissements d'enseignement et de recherche français ou étrangers, des laboratoires publics ou privés. 


\section{An inversion approach for analysing the physical properties of a \\ 2 seismic low-velocity layer in the upper mantle}

4 Jie Xiao ${ }^{a, b, c, *}$, Saswata Hier-Majumder ${ }^{b}$, Benoit Tauzin ${ }^{\text {d, e }}$, Dave Waltham ${ }^{\text {b }}$

5 (a) State Key Laboratory of Organic Geochemistry, Guangzhou Institute of

6 Geochemistry, Chinese Academy of Sciences, Guangzhou, 510640, China

7 (b) Department of Earth Sciences, Royal Holloway, University of London, Egham,

8 TW20 0EX, United Kingdom

9 (c) University of Chinese Academy of Sciences, Beijing 100049, China

10 (d) Université de Lyon, UCBL, ENS Lyon, CNRS, Laboratoire de Géologie de Lyon, Terre,

11 Planètes, Environnement, Villeurbanne, France

12 (e) Research School of Earth Sciences, Australian National University, Canberra, Australian Capital Territory 0200, Australia

$14 *$ Corresponding author (Jie.Xiao.2016@live.rhul.ac.uk)

\section{Abstract}

17 In this article, we propose a new inversion scheme to calculate the melt volume

18 fractions from observed seismic anomalies in a low-velocity layer (LVL) located atop

19 the mantle transition zone. Our method identifies the trade-offs in the seismic

20 signature caused by temperature, solid composition, melt volume fraction, and

21 dihedral angle at the solid-melt interface. Using the information derived from the

22 amplitude of $P$-to-S conversions beneath the western US, we show that the multiple 
23 permissible solutions for melt volume fractions are correlated to each other. An

24 existing solution can be directly transformed into a different solution whilst leaving the

25 model output unaltered. Hence, the additional solutions can be rapidly derived given

26 an initial solution. The calculation of multiple solutions reveals the universal properties

27 to the whole range of solutions. A regional-averaged melt volume fraction of at least

$280.5 \%$ exists in every solution. In addition, the mantle potential temperature in the

29 western US is broadly lower than $1550 \mathrm{~K}$ and the LVL in this region tends to be basaltic-

30 rich. Using these insights, it is possible to give firm statements on the LVL and the solid

31 mantle even though a unique interpretation does not exist.

33 Keywords: Shear wave, low-velocity layer, partial melting, inverse problem, non-

34 uniqueness

361 Introduction

37 The mantle transition zone (MTZ), marked by a drastic change in the physical

38 properties of the silicate mineral phases, plays a crucial role in the convective flow

39 within the mantle. Owing to the sharp changes in density and volatile storage capacity

40 across the boundaries of the MTZ, it can act as an impediment to mass transfer and

41 sights of partial melting (Bercovici and Karato, 2003; Morra et al., 2010). Indirect

42 evidence of mass transfer between subducting slabs and surrounding mantle are

43 obtained from the so-called 'superdeep diamonds' which bear geochemical signature

44 of oxygen and carbon isotopic ratios that can be generated by mixing between mantle 
and subducting slabs at these depths. Seismic observations also support the evidence

46 of partial melting atop the MTZ. A low-velocity layer (LVL) located at $\sim 350 \mathrm{~km}$ depth

47 has been identified just above the mantle transition zone in numerous regions around

48 the world, with lateral thickness from tens to over a few hundred kilometres (e.g. Song

49 et al., 2004; Gao et al., 2006; Courtier and Revenaugh, 2007; Schaeffer and Bostock,

50 2010; Huckfeldt et al., 2013). Characterized by $2-3 \%$ reductions in shear wave

51 velocities, the LVL is characterized by a sharp interface with the overlying mantle,

52 indicating the likely presence of a chemical anomaly, in particular partial melting.

53 However, quantifying the fraction of melt has remained challenging as the

54 environmental and chemical parameters, such as the mantle temperature, bulk solid

55 composition and melt geometry, are not clearly understood.

56 The 350-km LVL has been frequently interpreted as a seismic signature of a small

57 fraction of melt triggered by volatile elements released from subduction zones

58 (Revenaugh and Sipkin, 1994) or mantle plumes (Vinnik and Farra, 2007). Since melts,

59 characterized by zero shear modulus, disproportionately reduce shear wave velocities,

60 seismic anomalies with low velocities are often qualitatively attributed to melting.

61 Interpreting the origin of the seismic velocity anomalies in the LVL is complicated due

62 to the competing influence of several parameters. While an increase in the

63 temperature typically leads to seismic velocity reductions, the influence of bulk mantle

64 composition on seismic velocities varies with depth (Xu et al., 2008). The multiple

65 factors also likely affect each other. For instance, melting may leave a strong impact on

66 the bulk solid composition, in particular the amount of basalt. The residual anomaly, 
67 defined as the difference between the observed shear velocity and the reference

68 velocity, can be attributed to the presence of melting, and used as a basis for

69 calculating the volume fraction of melt in the LVL. Hence, calculation of the melt

70 fraction requires accurate estimation of the reference seismic velocities, i.e. velocities

71 in the absence of melting for given temperature and solid composition.

72 In addition, the non-uniqueness in the LVL interpretations arises from the fact

73 that, in a partially molten layer, the seismic velocity reductions depend on both the

74 melting extent and the microstructure of the melt-bearing aggregates (Mavko, 1980;

75 von Bargen and Waff, 1986; Takei, 1998, 2002). The dihedral angle (also known as

76 wetting angle) at the solid-melt interface, controls the geometry of the load-bearing

77 framework of partially molten rocks (Hier-Majumder and Abbott, 2010), trading off

78 with inferred melt volume fraction. The chemical composition is also found to play a

79 moderate role in reducing the seismic speeds (Wimert and Hier-Majumder, 2012; Hier-

80 Majumder et al., 2014), and may alter the dihedral angle (Yoshino et al., 2005). The

81 numerical experiment of (Hier-Majumder et al., 2014) indicated the difficulties in

82 distinguishing different types of melt from the seismic observations as the fraction of

83 melt is very small. These therefore lead to extra non-uniqueness in the interpretation

84 of seismic anomalies.

85 A number of previous studies mitigated the issue of competing influences by

86 carrying out computationally expensive brute-force search to create lookup tables for

87 inferred melt volume fractions corresponding to different controlling factors (e.g. Hier-

88 Majumder and Courtier, 2011; Hier-Majumder et al., 2014; Hier-Majumder and Tauzin, 
2017). While a brute-force search can produce a particular scenario of inversion,

90 application of the approach is unable to ascertain if alternative solutions exist in the

91 parameter space. Although, in principle, the entire range of solutions could be

92 discovered through repetitive use of the algorithm given different combinations of the

93 parameters, it fails to rigorously tackle the nature of variations in the inferred melt

94 volume fractions caused by changes in the other factors. Therefore, a new inversion

95 scheme is required to interpret these geophysical observations and to address the

96 theoretical drawback of previous studies.

97 Here we present a mathematical formulation that uses the implicit symmetry of a

98 petrologic model to understand the non-uniqueness in the melt fraction inference. The

99 results from our work provide a more reliable evaluation of the long-standing problem

100 in rock physics. The principle of symmetry has been successfully applied in a sequence

101 stratigraphic problem (Xiao and Waltham, 2019), showing that the whole set of

102 solutions are closely linked even when an inverse problem is non-linear. An existing

103 solution can be directly transformed into another solution that leaves modelling

104 products unchanged, in the same way that rotating a square by $90^{\circ}$ can produce an

105 identical geometry. In this way, the search for all possible solutions can begin with an

106 initial solution generated through standard inversion techniques. The application of

107 the symmetry method can then allow the additional solutions to be calculated from

108 the initial solution.

109 Embedded with a predictive forward model of shear-velocities, our inversion

110 scheme is used to revisit the nature of the $350-\mathrm{km} \mathrm{LVL} \mathrm{beneath} \mathrm{the} \mathrm{western} \mathrm{US.} \mathrm{The}$ 
111 seismically anomalous layer in this region has been reported underneath the Oregon-

112 Washington border (e.g. Song et al., 2004), the Yellowstone (e.g. Fee and Dueker,

113 2004; Jasbinsek and Dueker, 2007), the Northern Rocky Mountains (e.g. Jasbinsek and

114 Dueker, 2007; Zhang et al., 2018), the Colorado Plateau/Rio Grande Rift (e.g. Jasbinsek

115 et al., 2010), and California (e.g. Vinnik et al., 2010). Once the complete set of solutions

116 has been derived, the lowest and highest possible fractions of melt within the LVL can

117 be easily determined. As such, we can generate a robust statement on the partial

118 melting effect in the LVL that does not rely on assumed values of the other

119 parameters. The calculation can also offer more reliable information about the solid

120 mantle, such as the plausible ranges of temperature and basalt fraction. For example,

121 the estimates of melt content and associated parameters can be used to infer the

122 budget of volatile elements in the mantle and the excess temperature of the mantle

123 plumes beneath the region.

1252 The 350-km LVL beneath the western US

1262.1 Seismic observations

127 The seismic data used here are teleseismic $P$-to-S conversions recorded on

128 receiver functions from the Transportable Array of seismic stations in the western US

129 (fig. 1). The portion of the seismic network consists in 820 seismic stations. Shear wave

130 velocity contrasts at around $350 \mathrm{~km}$ have been derived for 583 sites over a $0.5^{\circ} \times 0.5^{\circ}$

131 grid in latitude and longitude. The seismically anomalous layer covers an area of $1.8 \times$

$13210^{6} \mathrm{~km}^{2}$, with lateral thickness from 25 to $90 \mathrm{~km}$ (Hier-Majumder and Tauzin, 2017). 
134 To invert the shear wave speeds in the LVL from the seismic observations, we

135 follow the computational approach outlined in Hier-Majumder et al. (2014). We use

136 the results of conversion amplitudes at the top and the base of the LVL to estimate the

137 velocity variations. To eliminate systematic variations in the amplitude of converted

138 arrivals caused by the acquisition geometry (seismic wave incidence), we normalize the

139 observed seismic amplitudes prior to computation. The normalized amplitude is

140 calculated from the ratio of amplitudes of arrivals converted at the top of the LVL over

141 arrivals converted at the olivine-wadsleyite mineralogical phase change at $410 \mathrm{~km}$

142 depth:

$$
R_{\text {norm }}=\frac{A_{\mathrm{LVL}}}{A_{410}}<0
$$

143 , where $A_{\mathrm{LVL}}$ is the frequency-averaged amplitude at the top of the LVL recorded at

144 each cell on the grid, and $A_{410}$ is the frequency-averaged amplitude at the $410-\mathrm{km}$

145 discontinuity in the same cell. Using the normalized $R_{\text {norm }}$, we then calculate the shear

146 wave velocity $\left(V_{\mathrm{S}}^{\mathrm{obs}}\right)$ at each location from the normalized contrast between the shear-

147 velocity immediately above the $350-\mathrm{km} \mathrm{LVL}\left(V_{\mathrm{S}}^{350}\right)$ and the velocity immediately below

148 the 410-km discontinuity $\left(V_{\mathrm{S}}^{410}\right)$ :

$$
V_{\mathrm{S}}^{\text {obs }}=V_{\mathrm{S}}^{350}\left(1+R_{\text {norm }} \frac{V_{\mathrm{S}}^{410}-V_{\mathrm{S}}^{350}}{V_{\mathrm{S}}^{350}}\right)
$$

149 We calculate $V_{\mathrm{S}}^{350}$ and $V_{\mathrm{S}}^{410}$ as the shear wave velocities at the depths of $350 \mathrm{~km}$ and

150410 km, respectively, from the Preliminary Reference Earth Model (PREM, Dziewonski 

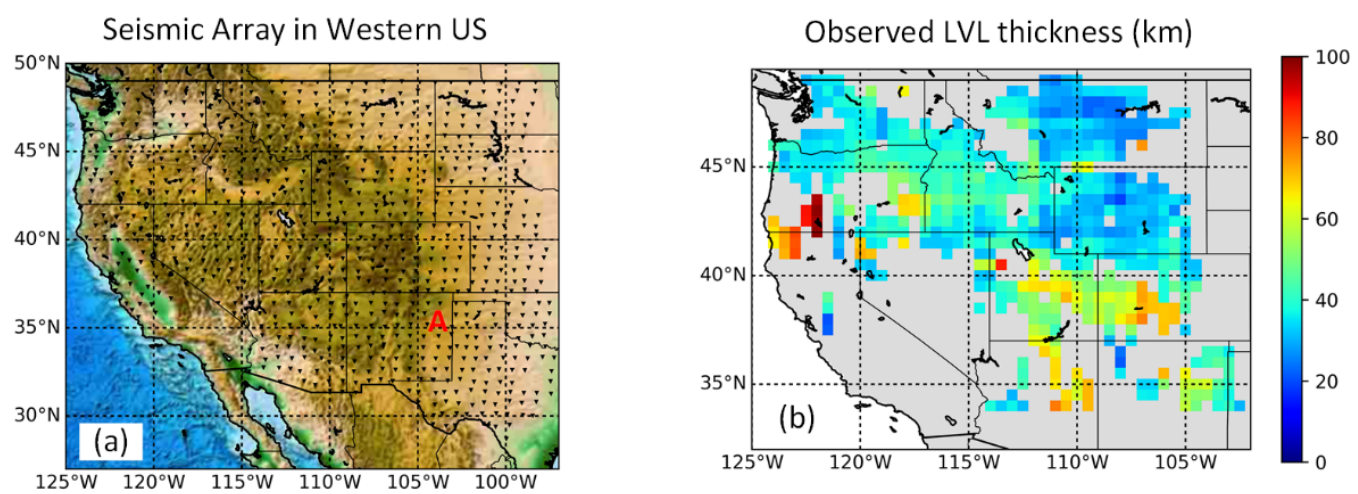

Normalized Amplitudes of $P$-to-S Conversions
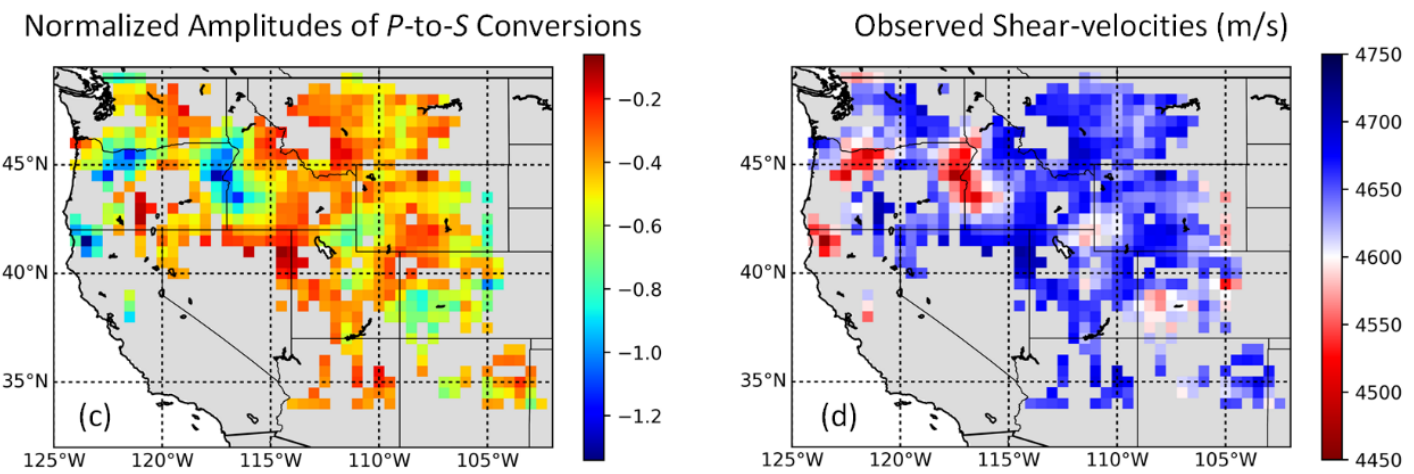

155 Figure 1 Seismic observations of the 350-km LVL below the western US. (a) A map of the dense

156 seismic array of 820 sites (black triangles, from Tauzin et al., 2013). The seismic cell (106.5 $5^{\circ}$,

$15738^{\circ} \mathrm{N}$ ) discussed later in this paper is labelled ' $A$ '. (b) The $350-\mathrm{km}$ LVL with lateral thickness is

158 identified beneath 583 sites (from Hier-Majumder and Tauzin, 2017). (c) Normalized amplitudes of

$159 P$-to-S converted arrivals. (d) Shear wave velocities in the LVL estimated from the seismic data

\subsection{Evaluating relative temperature variations}

We then evaluate the thermal variations in the LVL using the method outlined in

163 Tauzin and Ricard (2014). In this method, the relative temperature variations $(\Delta T)$ on

164 boundary topography are correlated with the relative thickness of the MTZ $(\delta h)$ and 
to the change in pressure at which the phase transition occurs) for the olivine-

170 (2014) calculated from the IASP91 spherical model of Kennett and Engdahl (1991). We

171 follow the empirical scheme of Tauzin and Ricard (2014) and set $\gamma_{410}=3.0 \mathrm{MPa} / \mathrm{K}$ and

$172 \gamma_{660}=0.64 \gamma_{410}-1.17$. The uncertainties associated with the supplementary

173 parameters will be discussed in later sections. Using these methods, we calculate the

174 temperature variations in the LVL from the MTZ thickness beneath the seismic array.
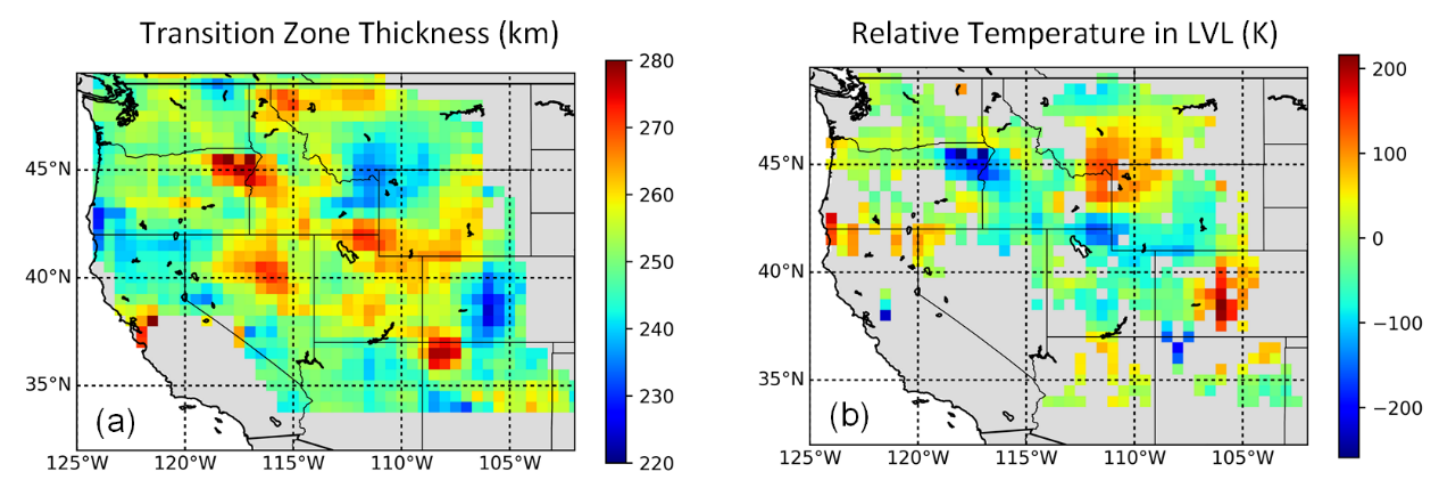

177 Figure 2 Thickness of MTZ (a) and relative temperature variations in the LVL (b) estimated from the

178 MTZ thickness (after Tauzin and Ricard, 2014)

\section{Forward modelling}

The forward model of shear-velocities presented here incorporates four primary 
184 wave speeds in the LVL consists of two independent phases. Firstly, we estimate the

185 reference velocities from the properties of the solid mantle. Secondly, we calculate the

186 changes in velocities as waves travelling through a melt-bearing aggregate using a

187 micromechanical model that involves both the fraction and geometry of the melt.

188

1893.1 Estimating reference velocities

190 We estimate the reference shear wave speeds in the solid mantle accounting for

191 the thermal and compositional properties of the mineral. The mantle temperature

192 below each site can be expressed as

$$
T=T_{0}+\frac{\mathrm{dT}}{\mathrm{dz}} z_{\mathrm{LVL}}+\Delta T
$$

193 , where $T_{0}$ is the potential temperature of the reference mantle, $\mathrm{d} T / \mathrm{d} z$ is the

194 adiabatic temperature gradient which is suggested as $0.4-0.5 \mathrm{~K} / \mathrm{km}$ in the upper

195 mantle (Katsura et al., 2010), $z_{\mathrm{LVL}}$ is the depth of $\mathrm{LVL}$ and $\Delta T$ is the relative

196 temperature variation at a given location. We set $z_{\mathrm{LVL}}$ at the average depth of $352 \mathrm{~km}$

197 as observed from the seismic profiles. To quantify the mantle composition, we follow

198 the definition from Xu et al. (2008) which parameterizes the solid bulk as a mechanical

199 mixture of mid-ocean ridge basalt and harzburgite. The composition of the solid

200 mantle can therefore be expressed as the volume fraction of basaltic component. We

201 can then formulate the reference shear wave velocities as $V_{\mathrm{S}}^{\text {ref }}=V_{\mathrm{S}}^{\text {ref }}\left(T_{0}, C\right)$, where

$202 T_{0}$ and $C$ are the potential temperature and basalt fraction of the mantle, respectively. 
204 database of Xu et al. (2008), in which seismic velocities are tabulated with associated

205 combinations of potential temperatures and basalt fractions. In the database,

206 potential temperatures range from 1000 to $2000 \mathrm{~K}$ with increments of $100 \mathrm{~K}$ whereas

207 basalt fractions range from 0 to $100 \%$ with increments of $5 \%$. While we can select a

208 given value of $C$ for the calculation, the temperature at any point on the seismic grid is

209 determined from the MTZ thickness as discussed above. As a result, we interpolate the

210 value of seismic velocity for the temperature evaluated at each location using a

211 second-order polynomial interpolation between two tabulated values. Using this

212 interpolation, we are able to calculate the value of reference shear wave speed at each

213 point for a given bulk basalt volume fraction and a given reference potential

214 temperature. Figure 3 presents the predictions of regional-average shear wave speeds

215 for a range of potential temperatures and basalt fractions at a constant pressure of

$21611.7 \mathrm{GPa}$. The thermal and compositional effects can trade off with each other and

217 thus different combinations of the two variables may lead to the same velocities. 


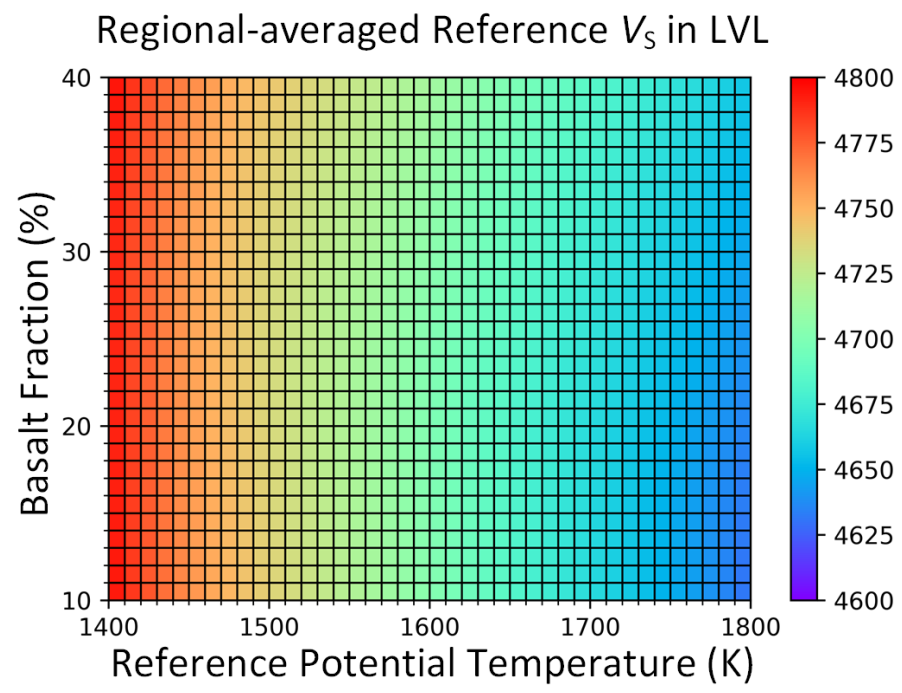

220 Figure $3 \mathrm{~A}$ heatmap showing the predicted regional-averaged reference shear wave velocities at

$221 \quad 11.7 \mathrm{GPa}$ in response to different combinations of reference potential temperature and basalt

222 fraction in the LVL beneath the western US

2243.2 Partial melting and velocity reductions

225 To simulate the influence of partial melting on seismic velocities, we employ the

226 modelling scheme of Takei (2002), where the shear wave speed variation $\xi$ is governed

227 by the effective elastic moduli of the aggregate:

$$
\xi=\sqrt{\frac{N / \mu}{\bar{\rho} / \rho_{\mathrm{s}}}}
$$

228 , where $N$ is the elastic modulus of the intergranular skeletal framework that indicates

229 the strength of contact between the neighbouring grains; $\mu$ is the shear modulus; $\rho_{s}$ is

230 the density of the solid bulk; and $\bar{\rho}$ is the volume-averaged density of the entire

231 aggregate which is calculated as:

$$
\bar{\rho}=\rho_{\mathrm{m}} \varphi+\rho_{\mathrm{s}}(1-\varphi)
$$


232, where $\rho_{m}$ is the density of the melt; $\varphi$ is the volume fraction of melt within the

233 aggregate.

234 In eq. 4, the elastic modulus $N$ is determined by both the melt volume fraction $\varphi$

235 and the contiguity ( $\psi$, i.e. the area fraction of the intergranular contact) of the melt:

$$
N=\mu(1-\varphi)\left[1-(1-\psi)^{n}\right]
$$

236 The contiguity $\psi$ depends on the melt volume fraction $\varphi$ and the dihedral angle $\theta$

237 between the solid grains and the melt; and $n$ is an exponent also depending on $\psi$

238 (Takei, 2002). The simulations of contiguity applied here are based on the micro-

239 structural model of von Bargen and Waff (1986) which formulates the contiguity $\psi$ as

240 the proportion that the contact area of grains occupy among the total contact area in a

241 partial molten aggregate:

$$
\psi=\frac{2 A_{\mathrm{gg}}}{2 A_{\mathrm{gg}}+A_{\mathrm{gm}}}
$$

242 , where $A_{\text {gg }}$ and $A_{\text {gm }}$ are the grain-grain contact area and grain-melt contact area per

243 unit volume, respectively. The values of $A_{\mathrm{gg}}$ and $A_{\mathrm{gm}}$ are calculated from the given

244 melt volume fraction and dihedral angle using polynomial functions:

$$
\left\{\begin{array}{c}
A_{\mathrm{gg}}=\pi-b_{\mathrm{gg}} \operatorname{power}\left(\varphi, p_{\mathrm{gg}}\right) \\
A_{\mathrm{gm}}=b_{\mathrm{gm}} \operatorname{power}\left(\varphi, p_{\mathrm{gm}}\right)
\end{array}\right.
$$

245 The required constants $b_{\mathrm{gg}}, b_{\mathrm{gm}}, p_{\mathrm{gg}}$ and $p_{\mathrm{gm}}$ are approximated from quadratic

246 polynomials of the dihedral angle (in degree), of which the values are outlined in von

247 Bargen and Waff (1986). Wimert and Hier-Majumder (2012) indicated this 
251 the melt volume fraction $\varphi$ and dihedral angle $\theta$, i.e. $\psi=\psi(\varphi, \theta)$. Moreover, shear

252 wave speed anomalies $\xi$ caused by partial melting can be formulated as a function

253 with respect to melt volume fraction and dihedral angle:

$$
\xi(\varphi, \theta)=\sqrt{\frac{(1-\varphi)\left[1-(1-\psi(\varphi, \theta))^{n}\right]}{1-\varphi\left(1-\rho_{\mathrm{m}} / \rho_{\mathrm{s}}\right)}}
$$

254 We estimate the densities of solid bulk $\rho_{\mathrm{s}}$ and melt $\rho_{\mathrm{m}}$ using the third-order Birch-

255 Murnaghan equation of state (EOS), as Ghosh et al. (2007) suggested for carbonated

256 peridotite melt. We implement the mathematic approximations using a Python

257 computational toolkit for microscale geodynamic study, named as Multiphase Material

258 Properties forward model (MuMaP, Hier-Majumder, 2017). The modelled shear wave

259 velocity reductions (in percentage) in response to a variety of melt volume fractions

260 and dihedral angles are illustrated in fig. 4. Each of the curves in the cross-plot

261 represents the shear wave velocity reductions caused by the melt with volume

262 fractions from 0 to $5 \%$ at a fixed dihedral angle, showing that the velocity in the

263 partially molten aggregates decreases rapidly as the fraction of melt increases. The

264 upward shifting of the curves indicates that, for the same melt volume fraction, a

265 smaller dihedral angle can result in greater reductions in the shear wave speed.

266 However, different combinations of dihedral angles and melt volume fractions may

267 produce the same extent of shear-velocity reduction. 


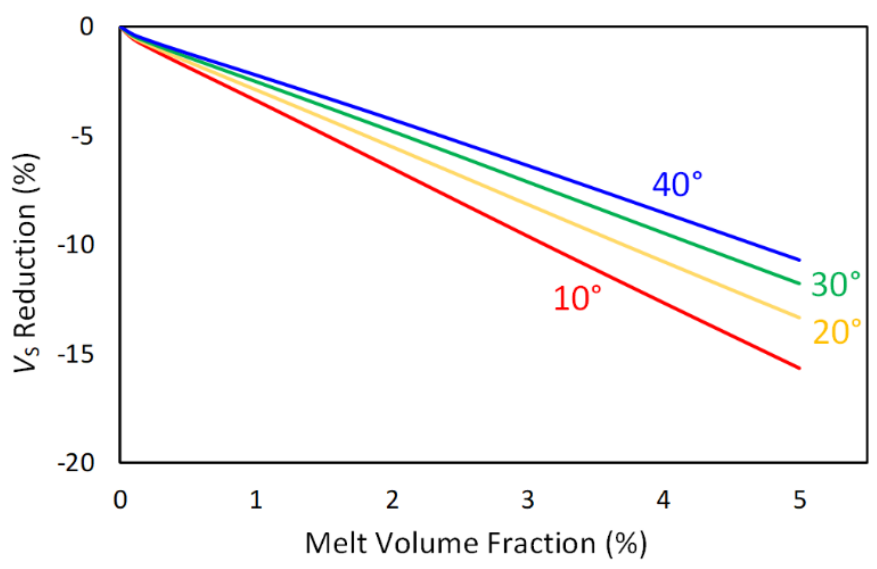

269 Figure 4 Predicted shear wave velocity reductions for different melt volume fractions and dihedral

270 angles. The corresponding dihedral angles to the curves are annotated on the plot. Each curve

271 shows the velocity reductions caused by changes in melt volume fraction at a fixed dihedral angle

\section{Model inversion}

274 The forward modelling approach described in the preceding section predicts the

275 shear wave velocity reductions in response to associated parameters. Alternatively, if

276 seismic data of the LVL are available, it is possible to calculate the velocity reductions

277 as a ratio of the observed velocity over the reference velocities:

$$
\xi=\frac{V_{\mathrm{S}}^{\mathrm{obs}}}{V_{\mathrm{S}}^{\mathrm{ref}}\left(T_{0}, C\right)}
$$

278 When embedded with an inversion scheme, the numerical model can be used to

279 deduce the multiple controls on seismic velocities. The inversion procedure can begin

280 with an initial solution that is built upon petrologic and seismological constraints. We

281 then investigate how to exploit and utilize the symmetry of the model, which can allow

282 us to alter the initial solution directly into another solution whilst giving the same 


\subsection{An initial solution based on a priori knowledge}

To incorporate the forward model and the observed data in a single framework,

288 we firstly combine eq. 9 and 10 :

$$
\sqrt{\frac{(1-\varphi) \cdot\left[1-(1-\psi(\varphi, \theta))^{n}\right]}{1-\varphi\left(1-\rho_{\mathrm{m}} / \rho_{\mathrm{s}}\right)}}=\frac{V_{\mathrm{S}}^{\mathrm{obs}}}{V_{\mathrm{S}}^{\mathrm{ref}}\left(T_{0}, C\right)}
$$

289 , which gives four unknowns (i.e. $T_{0}, C, \theta$ and $\varphi$ ) in one equation. To solve melt volume

290 fraction $\varphi$ from the eq. 11, the reference potential temperature $T_{0}$, basalt fraction $C$

291 and dihedral angle $\theta$ need to be specified. We initially assume the reference potential

292 temperature to be $1500 \mathrm{~K}$ in the region. We set the basalt fraction at $18 \%$, as

293 suggested in Xu et al. (2008) for common peridotite. The dihedral angle at the grain-

294 melt interface varies with the chemical composition of the melt. For example, Minarik

295 and Watson (1995) proposed dihedral angles varying from 25 to $30^{\circ}$ at the interface

296 between carbonate melt and molten aggregates; Mei et al. (2002) suggested a dihedral

297 angle of $28^{\circ}$ for molten aggregates with hydrous basalt melt. Here we initially assume a

298 dihedral angle of $\theta=25^{\circ}$. Given these a priori assumptions, $T_{0}, C$ and $\theta$ are specified,

299 and hence the melt volume fraction can be solved from eq. 11.

300 We then calculate the corresponding melt volume fraction $\varphi$ using a modified

301 Newton-Raphson root-search algorithm (Press et al., 2007, chap. 9.1), same as the

302 calculation in Hier-Majumder and Tauzin (2017). The algorithm begins with a bracket 
303 for the melt volume fraction between $1 \times 10^{-4} \%$ and $10 \%$ and iterates the searching

304 process until a convergence of $10^{-4} \%$ is achieved in the inferred fraction. Figure 5

305 shows the initial solution derived from the inversion using the seismic observations

306 and the constraints on $T_{0}, C$ and $\theta$. The melt fractions in the region vary spatially and

307 yields an average of $0.72 \%$. The synthetic velocities reproduced from the forward

308 model (fig. 5c) proves a good match to the real observations (fig. 1d).
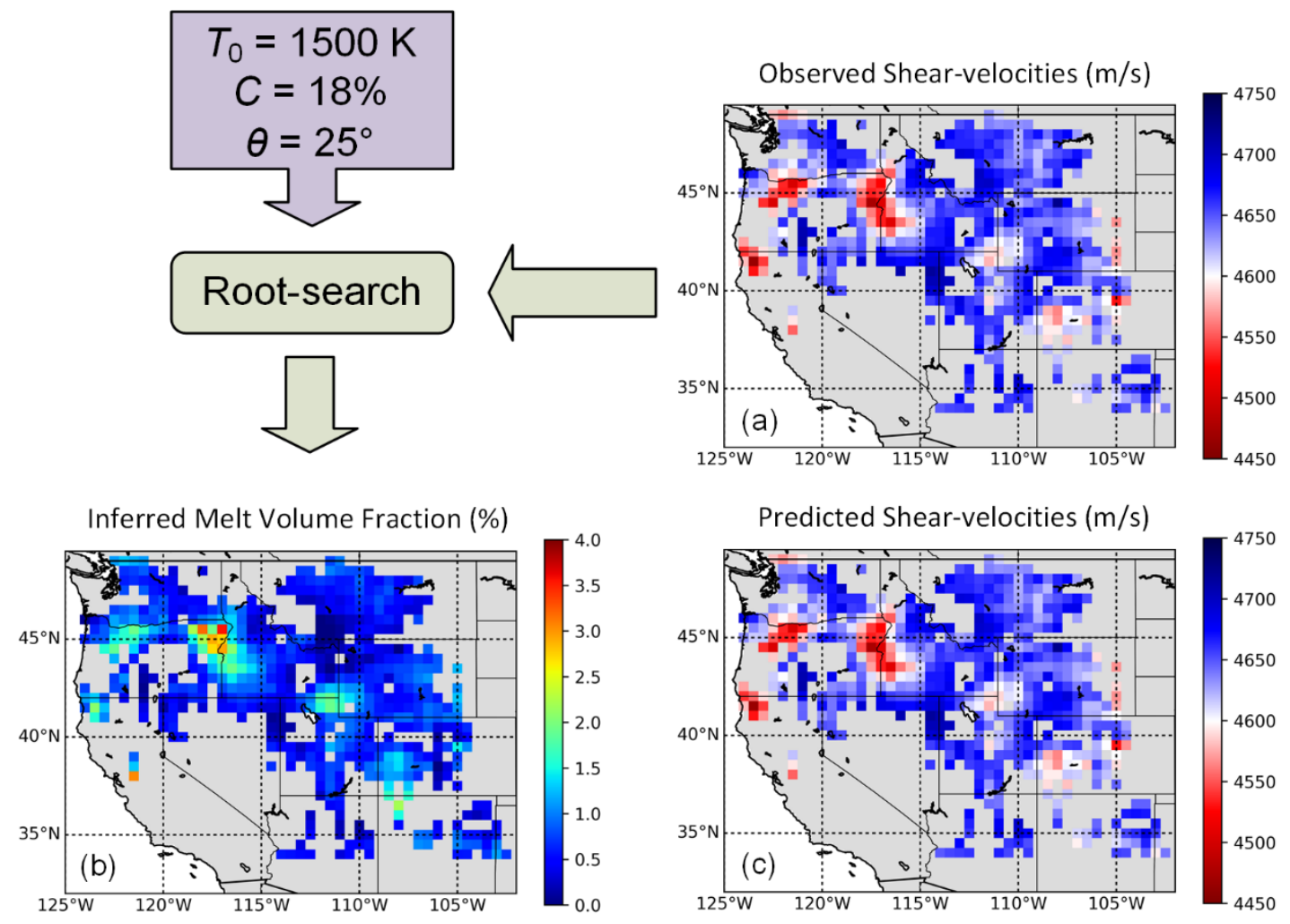

311 Figure 5 An initial solution found from the inverse problem. The observed shear wave velocities (a)

312 and reference values of $T_{0}, C$ and $\theta$ are used to provide constraints on the inversion. A particular

313 inference of the melt vol. \% within the LVL (b) is then generated using a root-search approach. The

314 regionally averaged fraction is calculated as $0.72 \%$ given $T_{0}=1500 \mathrm{~K}, C=18 \%$ and $\theta=25^{\circ}$. Using

315 the inferred melt vol. \% and the reference values, shear wave velocities (c) can be reproduced from 
The above calculation generates a single solution to the inverse problem. Since the

319 inverse problem is non-unique with respect to the input parameters $T_{0}, C$ and $\theta$, there

320 are, in principle, an infinite number of alternative solutions that can reproduce

321 identical seismic observations. Here we develop a quantitative approach to prove the

322 non-uniqueness and, more crucially, the transformation from an existing solution to an

323 alternative solution. The symmetry of the numerical model is found by properly

324 modifying the input parameters to obtain an unchanged output model. To start with,

325 we formulate the forward model of shear wave speed as:

$$
\boldsymbol{V}_{\mathrm{S}}=F\left(T_{0}, C, \theta, \boldsymbol{\varphi}\right)
$$

326 , where $\boldsymbol{V}_{\mathrm{S}}$ is the shear wave speeds in the LVL beneath the seismic sites; $F$ denotes a

327 general, non-linear function (in this work, $F$ is the forward model from the code

328 MuMaP_fwd) and $\varphi$ is a vector of melt volume fractions in the LVL. Note that 583

329 seismic sites are analysed in this study, and hence the vector lengths are 583 for both

$330 \boldsymbol{V}_{\mathrm{S}}$ and $\boldsymbol{\varphi}$. We then generate three perturbations $\delta T_{0}, \delta C$ and $\delta \theta$ respectively into the

331 three variables $T_{0}, C$ and $\theta$. These small changes in the model inputs thus give rise to

332 residuals in the modelled velocities, i.e. $\Delta \boldsymbol{V}_{\mathrm{s}}$. This can be written as:

$$
\Delta \boldsymbol{V}_{\mathrm{S}}=F\left(T_{0}+\delta T_{0}, C+\delta C, \theta+\delta \theta, \boldsymbol{\varphi}\right)-F\left(T_{0}, C, \theta, \boldsymbol{\varphi}\right)
$$

333 , which may be approximated in a linear form using the first-order Taylor's series:

$$
\frac{\partial F}{\partial T_{0}} \delta T_{0}+\frac{\partial F}{\partial C} \delta C+\frac{\partial F}{\partial \theta} \delta \theta=\Delta \boldsymbol{V}_{\mathrm{S}}
$$



, where $\partial F / \partial T_{0}, \partial F / \partial C$ and $\partial F / \partial \theta$ are finite derivatives of the function $F$ with respect to $T_{0}, C$ and $\theta$. We then calculate changes required in the melt volume

336 fractions (i.e. $\delta \varphi$ ) to compensate the changes in velocities resulting from the

337 perturbations. This can be expressed as:

$$
F\left(T_{0}+\delta T_{0}, C+\delta C, \theta+\delta \theta, \boldsymbol{\varphi}+\delta \boldsymbol{\varphi}\right)-F\left(T_{0}, C, \theta, \boldsymbol{\varphi}\right)=\mathbf{0}
$$

338 Approximation based on the Taylor's series gives:

$$
\frac{\partial F}{\partial T_{0}} \delta T_{0}+\frac{\partial F}{\partial C} \delta C+\frac{\partial F}{\partial \theta} \delta \theta+\frac{\partial F}{\partial \boldsymbol{\varphi}} \delta \boldsymbol{\varphi}=\mathbf{0}
$$

339 , where $\partial F / \partial \varphi$ is the finite derivative of the function $F$ with respect to $\varphi$. We then 340 solve $\delta \varphi$ by combining eq. 14 and 16:

$$
\delta \varphi=-\frac{\Delta V_{S}}{\partial F / \partial \varphi}
$$

341 In this equation, $\partial F / \partial \varphi$ can be calculated from the forward model. We make small

342 changes in $\boldsymbol{\varphi}$ and then run the model to predict the corresponding velocities. The

343 values of $\partial F / \partial \varphi$ are given by the difference in the modelling outputs divided by the

344 small changes in $\boldsymbol{\varphi}$. Note that $\delta \boldsymbol{\varphi}, \partial F / \partial \boldsymbol{\varphi}$ and $\Delta \boldsymbol{V}_{\mathrm{S}}$ are all vectors with a length of 583

345 as there are 583 locations in total. Given the perturbations $\delta T_{0}, \delta C$ and $\delta \theta$ and the

346 required adjustments in melt volume fractions $\delta \varphi$, the model $F\left(T_{0}+\delta T_{0}, C+\delta C, \theta+\right.$

$347 \delta \theta, \boldsymbol{\varphi}+\delta \boldsymbol{\varphi})$ can produce the same shear wave speeds as given by the initial solution.

348 The new solution can then be used as a basis for another transformation. Iterative

349 transformation can therefore derive all the additional solutions to the inverse problem. 
Using the forward model and symmetric transformation, we then examine the

353 entire parameter space and calculate alternative solutions. The parameter space can

354 be considered as a 3-D volume of which the three dimensions are potential

355 temperature $\left(T_{0}\right)$, basalt fraction $(C)$ and dihedral angle $(\theta)$. We define the ranges of

356 the parameters as 1400 to $1800 \mathrm{~K}$ in potential temperature, 10 to $40 \%$ in basalt

357 fraction and $10^{\circ}$ to $40^{\circ}$ in dihedral angle. We also set the increments at $10 \mathrm{~K}$ in

358 potential temperature, $1 \%$ in basalt fraction and $1^{\circ}$ in dihedral angle. Therefore, the

359 parameter space is finely sampled and thus the transformation approach can apply.

360 Each position in the parameter space can be described using the coordinates in the

361 three dimensions. If a solution exists in position $\left(T_{0}, C, \theta\right)$, then the corresponding melt

362 volume fraction vector can be written as $\varphi\left(T_{0}, C, \theta\right)$. Once a solution is found, the

363 solutions in neighbouring positions can also be determined. Because the

364 transformation can be applied both forward and backward, six neighbouring solutions

365 should be examined, including $\varphi\left(T_{0}+\delta T_{0}, C, \theta\right), \boldsymbol{\varphi}\left(T_{0}-\delta T_{0}, C, \theta\right), \boldsymbol{\varphi}\left(T_{0}, C+\delta C, \theta\right)$,

$366 \boldsymbol{\varphi}\left(T_{0}, C-\delta C, \theta\right), \boldsymbol{\varphi}\left(T_{0}, C, \theta+\delta \theta\right)$ and $\boldsymbol{\varphi}\left(T_{0}, C, \theta-\delta \theta\right)$. We calculate the additional

367 solutions through the following procedure:

368 (1) Create an empty list. Add the coordinate of the initial solution into the list.

369 (2) For each solution in the list, calculate the solutions in the neighbouring

370 positions that are inside of the parameter space but not existing in the list.

371 (3) Add the solutions found in step (2) into the list.

372 (4) Return to step (2) and repeat the workflow until no new solution can be 

added into the list.

$374 \quad$ Note that this is different from a brute-force search which involves a root-

375 searching approach for calculating the melt volume fraction beneath every location

376 given different combinations of $T_{0}, C$ and $\theta$. In contrast, the symmetric transformation

377 is straightforward as it can simultaneously derive the melt volume fraction beneath the

378 whole area. Since this method works directly on the behaviour of the solution with

379 respect to perturbations, it also allows us to predict regions where solution does not

380 exist and the solution containing the lowest possible average melt fractions, which was

381 intractable with the method described by Hier-Majumder et al. (2014).

4.4 Complete solutions to the inverse problem

Using the above computational procedure, we derive all the solutions in the

385 parameter space. All the possible solutions can reproduce the same synthetic shear

386 wave velocities from the forward model. Significant spatial variations in the inverted

387 melt volume are found in every solution. Because the melt volume fraction should

388 always be non-negative, the calculated vectors of $\varphi$ where one or more negative

389 values exist should be removed. Given this requirement, limits can be placed to bound

390 the symmetric transformation, i.e. not every combination of potential temperatures,

391 basalt fractions and dihedral angles in the parameter space is compatible with the

392 seismic observations, though not a unique solution to the inverse problem can be

393 found. Example of the variations in calculated melt volume fractions and the

394 transformation limits in the multiple controlling factors are demonstrated in fig. 6. 

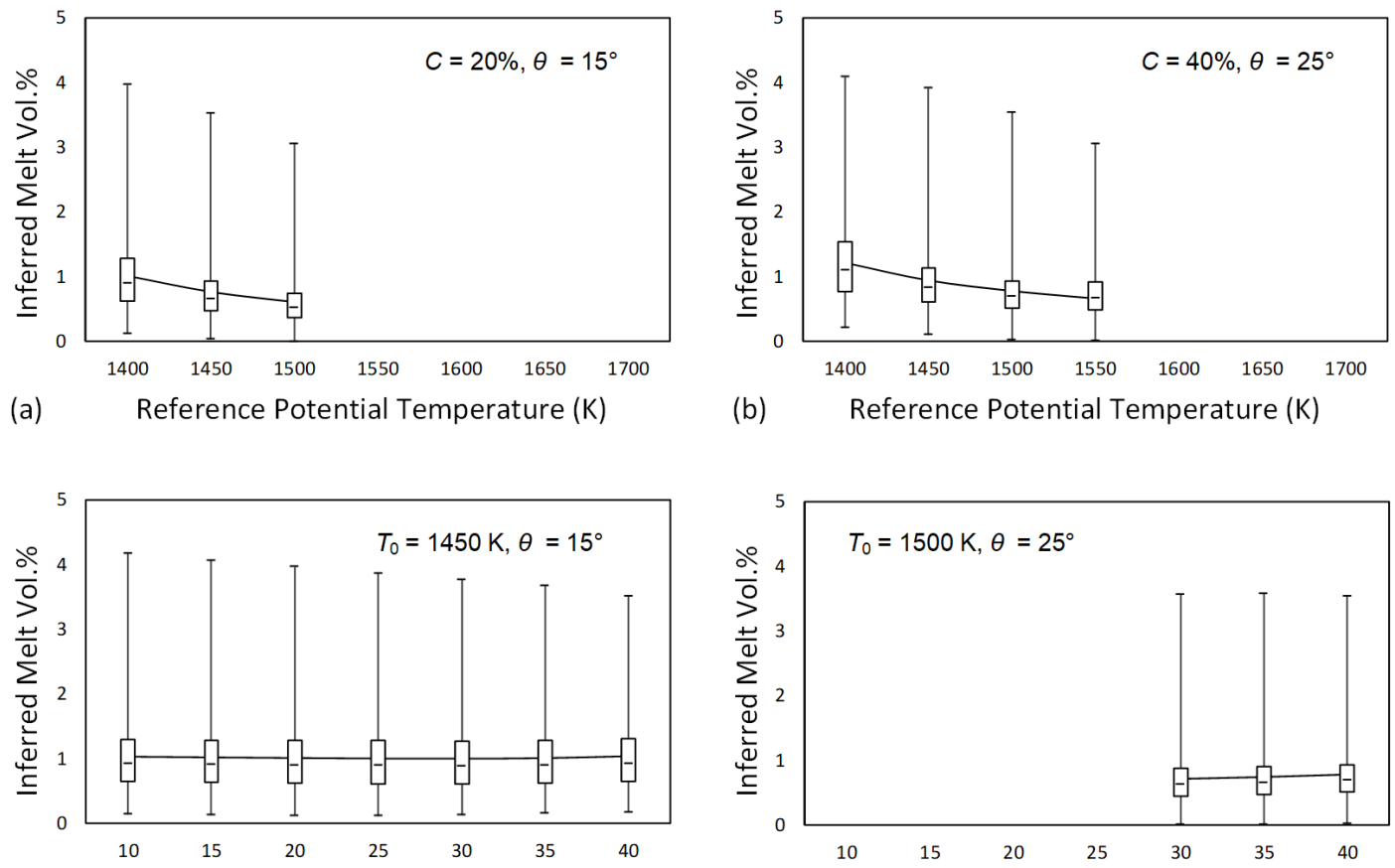

(c)

(d)

Basalt Fraction (\%)

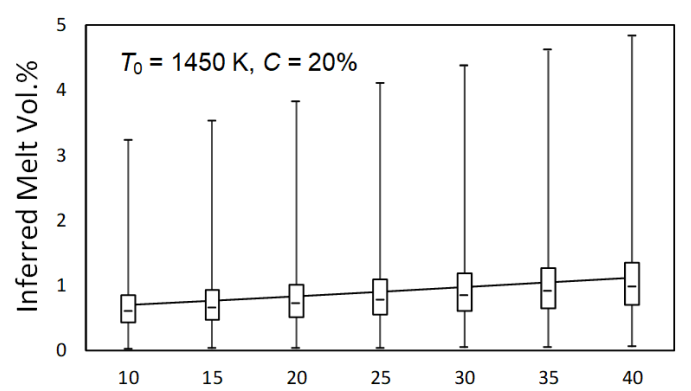

(e)

Dihedral Angle $\left({ }^{\circ}\right)$

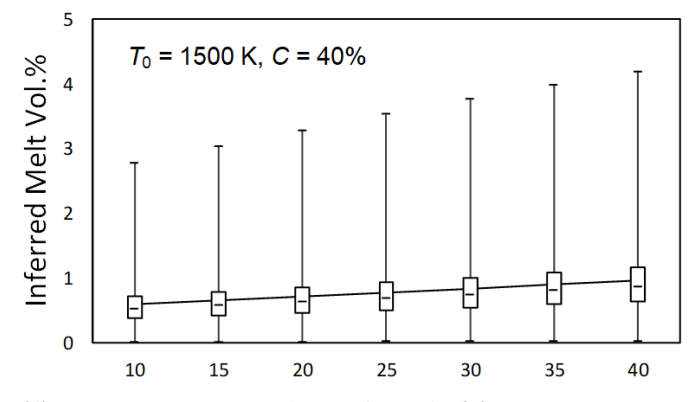

(f)

Dihedral Angle $\left({ }^{\circ}\right)$

396 Figure 6 Series of box-plots showing the estimated melt vol.\% beneath all locations with median

397 indicated by the horizontal line within each box, upper/lower quartiles indicated by the upper/lower edges of the box and maximum/minimum indicated by whiskers of the boxes. (a) \& (b) Inferred melt vol.\% as a function of reference potential temperature with fixed basalt fraction and dihedral angles. (c) \& (d) Inferred melt vol.\% as a function of basalt fractions with fixed reference potential temperature and dihedral angle. (e) \& (f) Inferred melt vol.\% as a function of dihedral angle with fixed reference potential temperature and basalt fractions. Note that no solution can be found given $T_{0} \geqslant 1500$ in (a), $T_{0} \geqslant 1550 \mathrm{~K}$ in (b) and $C \leqslant 30 \%$ in (d) 
406 lowest possible averaged melt volume fraction is $0.51 \%$, associated with $T_{0}=1550 \mathrm{~K}, C$

$407=40 \%$ and $\theta=10^{\circ}$. In this solution, the melting is not predicted beneath some regions,

408 for instance at the triple border between Idaho, Montana and Wyoming. Considering

409 the sharp boundary atop the LVL, this may just be an artefact because the variations in

410 solid bulk are unlikely to produce the rapid velocity reductions. However, this solution

411 is still meaningful since it places a lower-bound below the regional-averaged melt

412 volume fraction within the observed LVL. In contrast, the highest possible averaged

413 melt volume fraction that exists in the parameter space yields $1.47 \%$, associated with

$414 T_{0}=1400 \mathrm{~K}$, basalt fraction $C=10 \%$ and dihedral angel $\theta=10^{\circ}$, as shown in fig. 8.

415 Examples of the trade-offs between the estimated melt volume fraction below a given

416 location and the multiple controls are displayed in fig. 9 by cross-plotting the estimates

417 and the corresponding controlling factors. Whilst the forward model used here is non-

418 linear, application of the proposed method has indicated the trajectories that link

419 together the multiple solutions in the parameter space.

420 


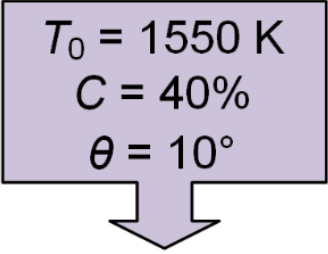

Inferred Melt Volume Fraction (\%)
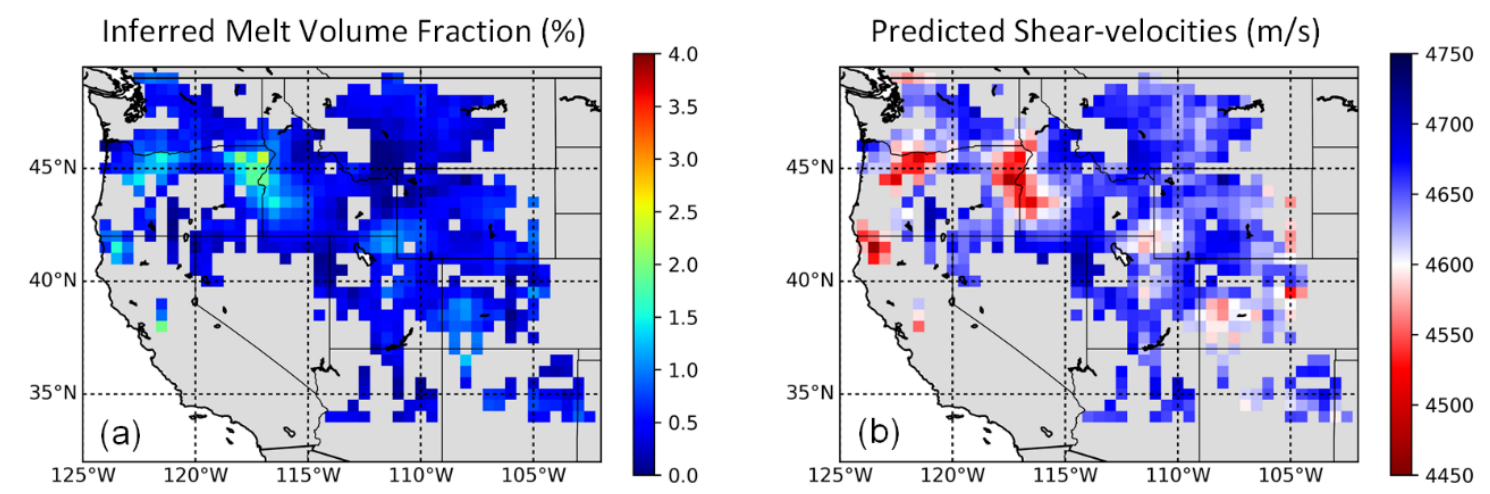

Figure 7 The end-member solution with the minimum melt vol. \% within the LVL beneath the w.

423 The regional averaged melt vol. $\%$ is $0.51 \%$ given $T_{0}=1550 \mathrm{~K}, C=40 \%$ and $\theta=10^{\circ}$. Note that this

424 solution is directly derived from the initial solution, rather than from a brute-force search
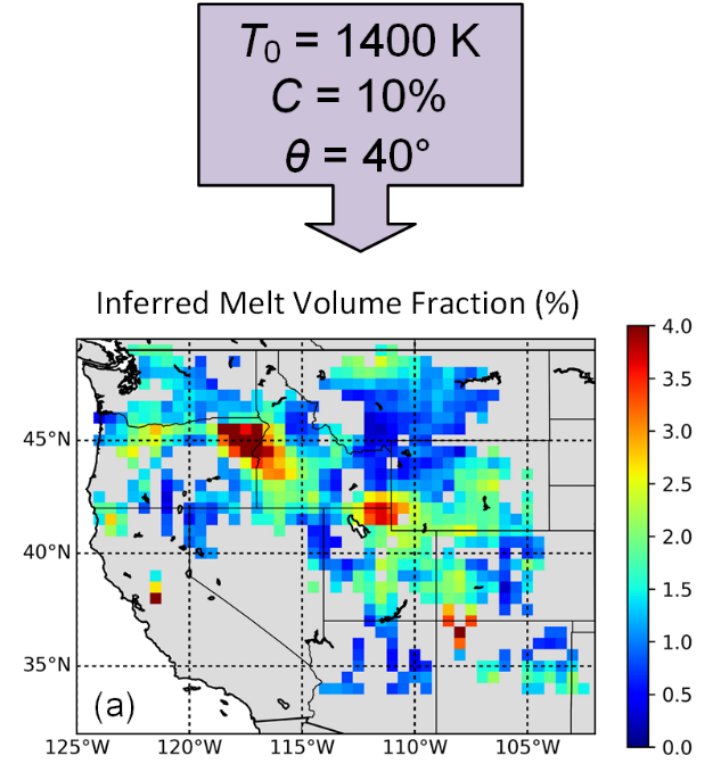

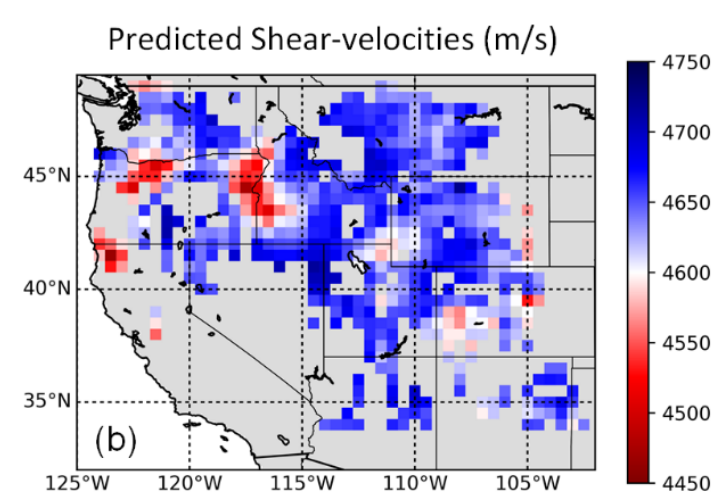

427 Figure 8 The end-member solution with the maximum melt vol. \% within the LVL beneath the

428 region. The regional averaged melt vol. $\%$ is $1.47 \%$ given $T_{0}=1400 \mathrm{~K}, C=10 \%$ and $\theta=40^{\circ}$. Note 

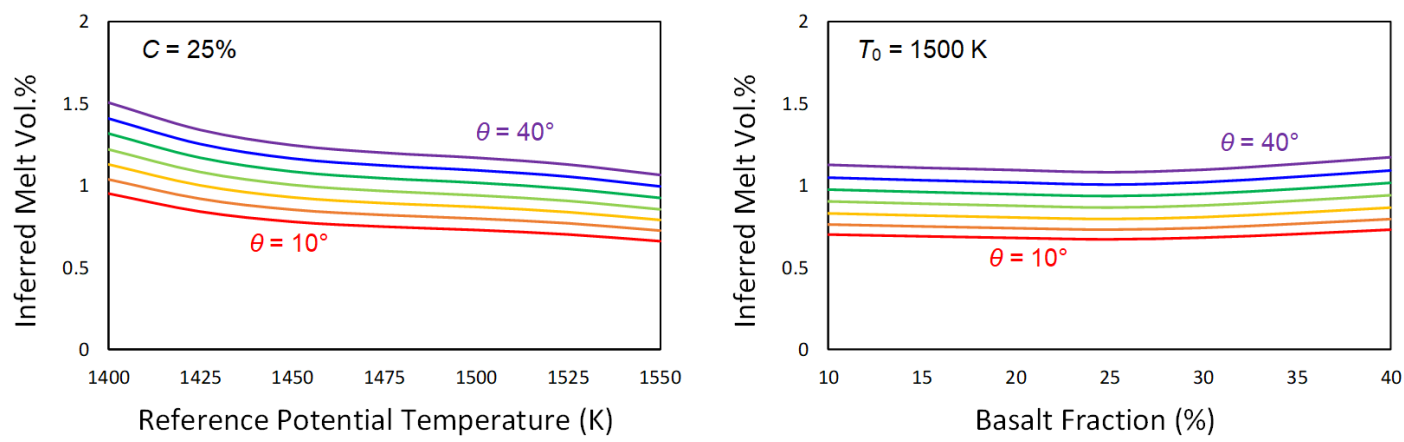

431 Figure 9 Cross-plots of inferred melt volume fraction beneath $106^{\circ} \mathrm{W}, 35^{\circ} \mathrm{N}$ (label A in fig. 1a)

432 versus (a) the reference potential temperature for different dihedral angles ranging from $10^{\circ}$ to $40^{\circ}$

433 (annotated on the plot) with constant intervals of $5^{\circ}$ given a fixed basalt composition and (b) the

434 basalt fraction in the bulk composition for a range of different dihedral angles given a fixed

435 potential temperature

\section{Discussion}

$438 \quad$ Using a numerical inversion approach, we have examined the LVL at $350 \mathrm{~km}$

439 underneath the western US. The shear-velocity anomalies and impedance contrasts in

440 this zone have been thought to indicate a small fraction of volatile-rich melt (Hier-

441 Majumder and Tauzin, 2017) released either by the decarbonation during the Farallon

442 slab subduction (Thomson et al., 2016) or by the dehydration from the upwelling of

443 the Yellowstone mantle plume or small-scale convection within the MTZ (Bercovici and

444 Karato, 2003; Richard and Bercovici, 2009; Zhang et al., 2018). Despite the presence of

445 petrological and geochemical evidences of melting near the MTZ, determination of the

446 quantity of melting from seismic signatures remains hard work owing to the trade-offs

447 that exist between various controlling factors. Due to the lack of geophysical and

448 geochemical constraints, it is difficult, if not impossible, to distinguish the individual 
449 effects of temperature, composition and partial melting. A recent study has further

450 suggested that these multiple controls are strongly correlated, leading to a

451 disagreement between the experimental measurements and theoretical estimates

452 (Freitas et al., 2019).

453 Our numerical scheme based on a symmetry is able to cover all solutions. Using a

454 forward model, we firstly generate an arbitrary solution assuming $T_{0}=1500 \mathrm{~K}, C=18 \%$

455 and $\theta=25^{\circ}$. This is a successful solution as the shear wave velocities it predicts are

456 consistent with the observations. The inverse problem is then linearized to find

457 neighbouring solutions to the initial solution. As the controlling parameters have only a

458 limited range of plausible values (in this work $1400 \leq T_{0} \leq 1800 \mathrm{~K}, 10 \% \leq C \leq 40 \%$ and

$\left.45910^{\circ} \leq \theta \leq 40^{\circ}\right)$, the symmetry gives a quasi-complete set of solutions subject to the

460 necessary constraint that the melt volume fraction in the upper mantle must always be

461 non-negative. This constraint can be justified as the effects of temperature and

462 composition are already taken into account. Given the above treatment, it is then a

463 simple matter to find the combinations of parameters that reveals the end-member

464 possibilities (e.g. maximum and minimum degrees of partial melting).

465 The modelling results show that a regional-averaged melt volume fraction of

466 approximately $0.51 \%$ is necessary to explain the sharp shear-velocity reductions at 350

$467 \mathrm{~km}$ beneath the western US. This is the minimum extent of melting required to

468 produce the observed LVL, whatever the solid mantle conditions and the geometry of

469 the melt are. As Hier-Majumder and Courtier (2011) suggested, at such a small level of

470 melting, a near neutrally buoyant melt can migrate over the LVL due to surface 
471 tension, whereas the drainage efficiency of both buoyant and dense melts is likely

472 insignificant.

473 As no solution has been found to be associated with a reference potential

474 temperature higher than $1550 \mathrm{~K}$, we can place an upper-bound on the variations in the

475 reference potential temperature. The modelling output also shows that the range of

476 variations in basalt fraction depends on the assumed reference potential temperature.

477 At a low reference potential temperature (e.g. $1400 \mathrm{~K}$ ), the basalt fraction may vary

478 from $10 \%$ to $40 \%$. In contrast, at a higher reference potential temperature, solutions

479 can only be in the basaltic-rich zone (e.g. fig. 6d). For instance, Hier-Majumder and

480 Tauzin (2017) estimated the reference potential temperature as approximately $1550 \mathrm{~K}$.

481 If this is the case, then we can make a statement that the basalt fraction in the LVL

482 beneath the western US is no less than $40 \%$. Hence, whilst the thermal and

483 compositional conditions are still under-constrained, our model work offers more

484 reliable information about the mantle physical properties.

485 In addition, our inverse method unravels trade-offs between parameters. As the

486 forward model is non-linear, there is no simple analytical tool for determining these

487 competing effects. The numerical approach proposed here allows estimating the rates

488 of change in the inferred melt volume fraction caused by changes in other parameters.

489 According to the modelling outputs, the significance of the trade-offs between inferred

490 melt volume fractions and other parameters can be summarized as:

491 (1) For a given dihedral angle and a given basalt fraction, the inferred melt volume fractions present a strong negative correlation with the assumed 
reference potential temperatures (fig. 6a \& b).

(2) For a given reference potential temperature and a given dihedral angle, the inferred melt volume fractions are insensitive to the assumed basalt fractions (fig. 6c \& d).

(3) For a given reference potential temperature and a given basalt fraction, the inferred melt volume fractions present a modest positive correlation with the In this analysis, a number of assumptions are made about the mantle: it is in a

501 state of chemical disequilibrium (described as a mechanical mixture of basalt and

502 harzburgite), melt films are not playing a role in velocity reduction, the transition zone

503 thickness reflects temperature variations, the Clapyron slope is known, and receiver

504 function estimates of MTZ thickness are accurate.

505 This study calculates the temperature variations from the thickness of the MTZ

506 using the empirical correlation proposed by Tauzin and Ricard (2014). The empirical

507 model relies on several assumptions, for example that only temperature varies MTZ

508 thickness and that no vertical variation occurs in temperature from the MTZ to the LVL.

509 As observed from tomographic models (with low vertical resolution), the MTZ has

510 consistent structures over the whole range of depth, in particular the stalled Juan de

511 Fuca/Farallon slab (Burdick et al., 2008; Schmandt et al., 2011; Hier-Majumder and

512 Tauzin, 2017). Although an entirely consistent MTZ should not be expected, dealing

513 with the absolute topography of discontinuities to infer the temperatures would likely 514 introduce more uncertainties, as would require a precise correction of the effect of 
515 shallow velocity heterogeneities from 3-D tomographic models, which have their own

516 limitations.

517 Another assumption involved here is that one can extract reliable MTZ thickness

518 from receiver functions, while interference effects from seismic phases that do not

519 interact with MTZ boundaries can be neglected. It has indeed been shown through

520 modeling that the effect of interfering phases is small (Tauzin et al., 2013). The move-

521 out of these phases is different from the one from direct conversions (Guan and Niu,

522 2017) and stacking along move-out curves for direct conversions is efficient in

523 removing their effect. Besides, slant-stack diagrams and slowness weighted stacking

524 (e.g. Guan and Niu, 2017; Hier-Majumder and Tauzin, 2017) show no evidence for

525 interference effects in several locations of the western US.

526 Apart from the primary controls on the seismic velocities we have investigated,

527 there are other factors that can influence the seismic wave speeds. In this work, the

528 Clapeyron slopes $\gamma$ are set to values from a compilation of experimentally obtained

529 values (Tauzin and Ricard, 2014). The values of matrix density $\rho_{\mathrm{s}}$ and melt density $\rho_{\mathrm{m}}$

530 are set to constants as suggested in previous studies. The thickness of MTZ applied

531 here are also from supplementary dataset whereas alternative empirical models (e.g.

532 Keifer and Dueker, 2019) would produce different scenarios. These additional

533 complications can lead to substantial uncertainties in estimated melt volume fractions.

534 However, the inversion technique presented here is independent from the forward

535 model and can easily be adapted to include these factors. While in this paper the

536 application of the inversion has been demonstrated using 1-D column simulation, the 
537 technique could be applied to more sophisticated models that are spatially 2-D or 3-D.

538 For future work, we intend to apply our modelling approach to investigating the LVL

539 identified in other regions that differ in tectonic settings, for example, the Hawaii

540 Islands (Huckfeldt et al., 2013) which are dominated by mantle plumes. An effort will

541 be done for accounting for uncertainties from seismic data and propagate it into

542 mantle melting estimates.

\section{Conclusions}

545 The interpretation of the observed seismic structures in the upper mantle, like

546 many other geophysical inverse problems, is hampered by the fundamental challenge

547 of non-uniqueness. In this work, we investigate the influence of thermal,

548 compositional and melting effects on the pervasive LVL at $350 \mathrm{~km}$ beneath the western

549 US. We develop an inversion scheme, based on the principle of symmetry, for

550 generating the full range of solutions in the parameter space. Although a unique

551 solution is not available, the calculation of an ensemble of solutions allows extracting

552 the properties that are common to all solutions. A key result of our inversion is that a

553 minimum fraction of $\sim 0.5 \%$ melt by volume is necessary to explain the seismically

554 anomalous layer in the region. The scheme also encapsulates the ranges of variations

555 in the thermal and compositional factors. Consequently, the application of the

556 proposed inversion technique can provide more robust interpretation of seismic

557 velocity reductions within the mantle. 
560 The authors would like to thank Editor Mark Jellinek and two anonymous reviewers for

561 their constructive comments. Jie Xiao acknowledges support from the Strategic Priority

562 Research Program of the Chinese Academy of Sciences (XDA14010103) and China

563 National Major S\&T Program (2017ZX05008-002-030). Jie Xiao also wishes to thank

564 China Scholarship Council (CSC) for funding his PhD research. This research is also

565 supported by the European Union's Horizon 2020 research and innovation programme

566 under Benoit Tauzin's Marie Sklodowska-Curie grant agreement 793824.

568 References

569 Bercovici, D., and Karato, S.-i., 2003, Whole-mantle convection and the transition-zone $570 \quad$ water filter: Nature, v. 425, no. 6953, p. 39.

571 Burdick, S., Li, C., Martynov, V., Cox, T., Eakins, J., Mulder, T., Astiz, L., Vernon, F. L., Pavlis, G. L., and van der Hilst, R. D., 2008, Upper mantle heterogeneity beneath North America from travel time tomography with global and USArray transportable array data: Seismological Research Letters, v. 79, no. 3, p. 384-

576 Courtier, A. M., and Revenaugh, J., 2007, Deep upper-mantle melting beneath the Tasman and Coral Seas detected with multiple ScS reverberations: Earth and Planetary Science Letters, v. 259, no. 1-2, p. 66-76.

579 Fee, D., and Dueker, K., 2004, Mantle transition zone topography and structure beneath the Yellowstone hotspot: Geophysical Research Letters, v. 31, no. 18. 
581 Freitas, D., Manthilake, G., Chantel, J., Bouhifd, M., and Andrault, D., 2019,

582 Simultaneous measurements of electrical conductivity and seismic wave

583 velocity of partially molten geological materials: effect of evolving melt texture:

$584 \quad$ Physics and Chemistry of Minerals, p. 1-17.

585 Gao, W., Matzel, E., and Grand, S. P., 2006, Upper mantle seismic structure beneath eastern Mexico determined from $\mathrm{P}$ and $\mathrm{S}$ waveform inversion and its implications: Journal of Geophysical Research: Solid Earth, v. 111, no. B8.

Ghosh, S., Ohtani, E., Litasov, K., Suzuki, A., and Sakamaki, T., 2007, Stability of carbonated magmas at the base of the Earth's upper mantle: Geophysical research letters, v. 34, no. 22.

Guan, Z., and Niu, F., 2017, An investigation on slowness-weighted CCP stacking and its application to receiver function imaging: Geophysical Research Letters, v. 44, no. 12 , p. $6030-6038$.

594 Hier-Majumder, S., 2017, MuMaP_fwd, Version: 1.0, Zenodo, http://doi.org/10.5281/zenodo.1040971.

596 Hier-Majumder, S., and Abbott, M. E., 2010, Influence of dihedral angle on the seismic velocities in partially molten rocks: Earth and Planetary Science Letters, v. 299, no. $1-2$, p. 23-32.

599 Hier-Majumder, S., and Courtier, A., 2011, Seismic signature of small melt fraction atop 600 the transition zone: Earth and Planetary Science Letters, v. 308, no. 3-4, p. 334601 342. 
602

603

604

605

606

607

608

609

610

611

612

613

614

615

616

617

619

620

621

622

Hier-Majumder, S., Keel, E. B., and Courtier, A. M., 2014, The influence of temperature, bulk composition, and melting on the seismic signature of the low-velocity layer above the transition zone: Journal of Geophysical Research: Solid Earth, v. 119, no. 2 , p. 971-983.

Hier-Majumder, S., and Tauzin, B., 2017, Pervasive upper mantle melting beneath the western US: Earth and Planetary Science Letters, v. 463, p. 25-35.

Huckfeldt, M., Courtier, A. M., and Leahy, G. M., 2013, Implications for the origin of Hawaiian volcanism from a converted wave analysis of the mantle transition zone: Earth and Planetary Science Letters, v. 373, p. 194-204.

Jasbinsek, J., and Dueker, K., 2007, Ubiquitous low-velocity layer atop the 410-km discontinuity in the northern Rocky Mountains: Geochemistry, Geophysics, Geosystems, v. 8, no. 10.

Jasbinsek, J. J., Dueker, K. G., and Hansen, S. M., 2010, Characterizing the $410 \mathrm{~km}$ discontinuity low-velocity layer beneath the LA RISTRA array in the North American Southwest: Geochemistry, Geophysics, Geosystems, v. 11, no. 3.

Katsura, T., Yoneda, A., Yamazaki, D., Yoshino, T., and Ito, E., 2010, Adiabatic temperature profile in the mantle: Physics of the Earth and Planetary Interiors, v. 183 , no. $1-2$, p. $212-218$.

Keifer, I., and Dueker, K., 2019, Testing the hypothesis that temperature modulates 410 and 660 discontinuity topography beneath the eastern United States: Earth and Planetary Science Letters, v. 524, p. 115723. 
623 Kennett, B., and Engdahl, E., 1991, Traveltimes for global earthquake location and 624 phase identification: Geophysical Journal International, v. 105, no. 2, p. 429625465.

626 Mavko, G. M., 1980, Velocity and attenuation in partially molten rocks: Journal of Geophysical Research: Solid Earth, v. 85, no. B10, p. 5173-5189.

628 Mei, S., Bai, W., Hiraga, T., and Kohlstedt, D., 2002, Influence of melt on the creep behavior of olivine-basalt aggregates under hydrous conditions: Earth and

631 Minarik, W. G., and Watson, E. B., 1995, Interconnectivity of carbonate melt at low melt fraction: Earth and Planetary Science Letters, v. 133, no. 3-4, p. 423-437.

633 Morra, G., Yuen, D. A., Boschi, L., Chatelain, P., Koumoutsakos, P., and Tackley, P., 6342010 , The fate of the slabs interacting with a density/viscosity hill in the midmantle: Physics of the Earth and Planetary Interiors, v. 180, no. 3-4, p. 271-282.

636 Press, W. H., Teukolsky, S. A., Vetterling, W. T., and Flannery, B. P., 2007, Numerical recipes 3rd edition: The art of scientific computing, Cambridge university press, p. 445. mantle discontinuity: Nature, v. 369, no. 6480, p. 474.

641 Richard, G. C., and Bercovici, D., 2009, Water-induced convection in the Earth's mantle transition zone: Journal of Geophysical Research: Solid Earth, v. 114, no. B1. 
643 Schaeffer, A., and Bostock, M., 2010, A low-velocity zone atop the transition zone in 644 northwestern Canada: Journal of Geophysical Research: Solid Earth, v. 115, no.

$645 \quad$ B6.

646 Schmandt, B., Dueker, K., Hansen, S., Jasbinsek, J. J., and Zhang, Z., 2011, A sporadic

647 low-velocity layer atop the western US mantle transition zone and short-

648 wavelength variations in transition zone discontinuities: Geochemistry,

649 Geophysics, Geosystems, v. 12, no. 8.

650 Song, T.-R. A., Helmberger, D. V., and Grand, S. P., 2004, Low-velocity zone atop the $651410-\mathrm{km}$ seismic discontinuity in the northwestern United States: Nature, v. 427, 652 no. 6974, p. 530.

653 Takei, Y., 1998, Constitutive mechanical relations of solid-liquid composites in terms of 654 grain-boundary contiguity: Journal of Geophysical Research: Solid Earth, v. 103, 655 no. B8, p. 18183-18203.

656 Takei, Y., 2002, Effect of pore geometry on Vp/Vs: From equilibrium geometry to crack: Journal of Geophysical Research: Solid Earth, v. 107, p. 2043.

658 Tauzin, B., and Ricard, Y., 2014, Seismically deduced thermodynamics phase diagrams for the mantle transition zone: Earth and Planetary Science Letters, v. 401, p. 337-346.

661 Tauzin, B., Van Der Hilst, R. D., Wittlinger, G., and Ricard, Y., 2013, Multiple transition zone seismic discontinuities and low velocity layers below western United States: Journal of Geophysical Research: Solid Earth, v. 118, no. 5, p. 23072322. 
665

666

667

668

669

670

671

672

673

674

675

676 Wimert, J., and Hier-Majumder, S., 2012, A three-dimensional microgeodynamic model

677

678

679

680

681

682

683

684

Thomson, A. R., Walter, M. J., Kohn, S. C., and Brooker, R. A., 2016, Slab melting as a barrier to deep carbon subduction: Nature, v. 529, no. 7584, p. 76.

Vinnik, L., and Farra, V., 2007, Low S velocity atop the 410-km discontinuity and mantle plumes: Earth and Planetary Science Letters, v. 262, no. 3-4, p. 398-412.

Vinnik, L., Ren, Y., Stutzmann, E., Farra, V., and Kiselev, S., 2010, Observations of S410p and S350p phases at seismograph stations in California: Journal of Geophysical Research: Solid Earth, v. 115, no. B5.

von Bargen, N., and Waff, H. S., 1986, Permeabilities, interfacial areas and curvatures of partially molten systems: results of numerical computations of equilibrium microstructures: Journal of Geophysical Research: Solid Earth, v. 91, no. B9, p. 9261-9276. of melt geometry in the Earth's deep interior: Journal of Geophysical Research: Solid Earth, v. 117, no. B4.

Xiao, J., and Waltham, D., 2019, Non-uniqueness and symmetry in stratigraphic interpretations: A quantitative approach for determining stratal controls: Sedimentology, v. 66 , no. 5 , p. $1700-1715$.

Xu, W., Lithgow-Bertelloni, C., Stixrude, L., and Ritsema, J., 2008, The effect of bulk composition and temperature on mantle seismic structure: Earth and Planetary Science Letters, v. 275, no. 1-2, p. 70-79. 
685 Yoshino, T., Takei, Y., Wark, D. A., and Watson, E. B., 2005, Grain boundary wetness of 686 texturally equilibrated rocks, with implications for seismic properties of the upper mantle: Journal of Geophysical Research: Solid Earth, v. 110, no. B8.

688 Zhang, Z., Dueker, K. G., and Huang, H.-H., 2018, Ps mantle transition zone imaging 689 beneath the Colorado Rocky Mountains: Evidence for an upwelling hydrous mantle: Earth and Planetary Science Letters, v. 492, p. 197-205.

691 